\title{
Impact of Different Shelter Designs on Selected Serum Enzyme Activities and on Concentration of Stress Hormones in Dairy Cattle of Tamil Nadu, India
}

\author{
A. Paramasivam, P.S.L. Sesh", T. Sivakumar, P. Tensingh Gnanaraj and A. Thangavel
}

Department of Livestock Production and Management, Madras Veterinary College, Chennai, Tamil Nadu Veterinary and Animal Sciences University, Tamil Nadu-600 007, India

*Corresponding author

\section{A B S T R A C T}

\begin{tabular}{|l|}
\hline Ke y w o r d s \\
Shelter designs, \\
$\begin{array}{l}\text { Liver enzymes, } \\
\text { Stress hormones, } \\
\text { Dairy cattle }\end{array}$ \\
\hline Article Info \\
\hline $\begin{array}{l}\text { Accepted: } \\
\text { 10 December } 2017 \\
\text { Available Online: } \\
\text { 10 January } 2018\end{array}$ \\
\hline
\end{tabular}

\section{Introduction}

In Tamil Nadu, livestock sector contributes about 2.74 per cent of Gross State Domestic Product and the annual milk production in the state during the year 2015-16 was 7244 thousand tonnes. The state contributes about 4.65 per cent of milk production to the national milk grid and stands $9^{\text {th }}$ in milk production in the country (2015-16, NDDB Report).

\begin{abstract}
A study was carried out to assess the impact of shelter designs on the production performance of dairy cattle in the three selected agro - climatic zones of Tamil Nadu. viz., Southern, Cauvery delta and High rainfall zones. The effect of various shelter designs on the activity of liver enzymes and stress hormones were analyzed. The serum samples were collected throughout the year i.e. in all the four seasons, viz., winter, summer, south-west monsoon and north-east monsoon. For each season, a total of thirty samples (in duplicate) were collected from five housing types each comprising of six individual cattle from the three agro-climatic regions for measuring the bio-chemical profile. The activity of the four serum enzymes, namely AST, ALT, ChE and LDH were estimated spectrophotometrically and the three stress hormones namely cortisol, $\mathrm{T}_{3}$ and $\mathrm{T}_{4}$ were estimated by ELISA. The levels of AST, ALT, CHE and LDH differed significantly $(\mathrm{P}<0.01)$ between seasons in all the zones, Similarly a significant difference $(\mathrm{P}<0.01)$ in the concentration of cortisol, $\mathrm{T}_{3}$ and $\mathrm{T}_{4}$ was also observed between seasons in all the zones, whereas significant difference in $\mathrm{T}_{4}$ levels was observed between housing types in Southern zone only. Factor analysis study of this biochemical profile with other climatic, physiological and production parameters in all the three zones revealed that in Southern and Cauvery delta zones open housing is best and in High rainfall zone thatched roof system is the best.
\end{abstract}

Dairy farming is perceived by the farmers of our country as a part of an integrated agricultural system, where dairy and agriculture complement each other. In Dairying, housing management along with feeding plays a very important role in exploiting the genetic potential of dairy cows. Optimal feeding and housing are the prerequisite factor for milk production. To utilize the feed more efficiently the housing management becomes important which helps 
to maintain the thermo neutral zone in which the animals are most productive. The environmental conditions of livestock buildings significantly affect animal welfare and productivity.

The cow shelters under Indian conditions are designed to reduce the heat load because heat stress causes more stress to animals compared to winter. Principles for creating an optimum micro environment in and around the sheds are aimed to reduce heat gain and promote heat losses. In order to ensure optimum productivity of the dairy cattle, understanding of the existing housing patterns and the environmental stress in various agro-climatic regions is very essential. The most important environmental interventions done in recent days are those that have been done in housing and other attempts to ameliorate the thermal extremes. An environment in which stress is minimized would likely be favourable for efficient production of products derived from domestic farm animals and for ensuring the well-being of those species.

Hence a study was carried out to assess the impact of shelter designs on the production performance of dairy cattle in the three selected agro - climatic zones of Tamil Nadu viz. Southern, Cauvery delta and High rainfall zones. In this communication, the effect of various shelter designs on the activity of liver enzymes and stress hormones like cortisol / thyroid hormones are discussed.

\section{Materials and Methods}

The research work was carried out in two phases. The first one involved the base line survey to identify the existing dairy cattle housing system along with management practices followed by farmers in the representative districts from Southern (Madurai, Pudukkottai and Dindigul, Virudhunagar and Tirunelveli Districts),
Cauvery delta (Trichy and Thanjavur Dist.) and High rainfall zones (Kanyakumari Dist.).

The second one involved the seasonal stress assessment of the cattle in the selected farms from each zone, throughout the year i.e. all the four seasons, viz., winter, summer, south-west monsoon and north-east monsoon.

\section{Collection of samples}

For each season, a total of thirty samples (in duplicate) were collected from five housing types (open housing, thatch roofed, asbestos roofed, tile roofed and metal sheet housing), each comprising of six individual cattle for measuring the bio-chemical profile i.e. serum enzyme and hormone estimation from the three agro-climatic region. Thus, a total of 90 samples were collected during each season with a grand total of 360 samples (i.e. $30 \times 3 \times$ $4=360$ ) during the whole experimental period of one year. In each sampling season the number of animals sampled in each group was always greater than the five recommended by Whitaker (2000).

Whole blood was collected between 12.00 noon to $2.00 \mathrm{pm}$ from each cow through jugular vein puncture into tubes without EDTA for biochemical analyses as described by Grunwaldt et al., (2005). After clotting the blood, the tubes were centrifuged (1000 $\mathrm{g}$ for $30 \mathrm{~min}$ ) and required serum was immediately subjected to enzyme analysis and the remnant serum samples were stored at $-36^{\circ} \mathrm{C}$ for subsequent hormonal analysis.

\section{Serum enzymes}

The serum was examined for the following enzymes using an auto clinical chemistry analyzer (Biosystems) by standard methods described by Bergmeyar (1983) with the available commercial kits (AGAPPE Diagnostics Ltd., Ernakulam). 
Aspartate aminotransferase - (AST)

Alanine aminotransferase - (ALT)

Choline esterase - $(\mathrm{ChE})$

Lactate dehydrogenase - (LDH)

\section{Serum hormones}

The serum was examined for Cortisol, Triiodothyronine $\left(\mathrm{T}_{3}\right)$ and Thyroxine $\left(\mathrm{T}_{4}\right)$ hormones by enzyme immunoassay using microwell reader ( $\mu$ Quant, BioTek, USA) by standard methods described by Bergmeyar (1983) and Bhooshan et al., (2010) with the available commercial kits (BIOTRON diagnostic Inc. California).

\section{Statistical analysis}

The experimental data were statistically analyzed by using IBM SPSS Statistics Version 20.0 by using statistical tools two way analysis using General Linear Model Univariate Procedure. The significance was tested by using Tukeys HSD and T Test.

\section{Results and Discussion}

\section{Serum enzymes}

Activity of the four enzymes viz. AST, ALT, $\mathrm{ChE}$ and $\mathrm{LDH}$ have been determined in serum of animals under various housing types in all the four seasons of the three agro-climatic zones under study. The results of AST and ALT are presented in Table 1 and those of $\mathrm{ChE}$ and LDH are presented in Table 2.

\section{Aspartate Aminotransferase (AST)}

Activity of Aspartate aminotransferase (AST) enzyme was found to differ significantly $(\mathrm{P}<0.01)$ between seasons in all the zones. There was significant difference $(\mathrm{P}<0.01)$ between the housing systems in Cauvery delta zone. There was no significant difference in the interaction between the season and housing systems in all the zones. Highest value of AST (U/L) was found in thatched housing in High rainfall zone (119.4 \pm 21.1 U/L) during winter and the lowest under asbestos housing in High rainfall zone (54.1 $\pm 6.0 \mathrm{U} / \mathrm{L})$ during summer. The observed total mean value and observed higher value of AST falls within the normal range (78-132 U/1.). Similar kind of findings were reported by Srikandakumar and Johnson (2004); they observed in Australia that during winter higher AST level was recorded in Holstein and Australian milking Zebu and the values were 66.75 U/L and 98.25 U/L respectively.

\section{Alanine Aminotransferase (ALT)}

Activity of Alanine aminotransferase (ALT) enzyme was found to differ significantly $(\mathrm{P}<0.01)$ between seasons in all the zones. There was significant difference $(\mathrm{P}<0.01)$ between the housing systems in Southern zone alone. There was no significant difference in the interaction between the season and housing systems in all zones. Highest value of ALT (U/L) was found in tiled housing in High rainfall zone $(39.5 \pm 3.7 \mathrm{U} / \mathrm{L})$ during winter and the lowest under thatched housing in High rainfall zone $(18.0 \pm 3.1 \mathrm{U} / \mathrm{L})$ during summer.

The total mean values of ALT for all housing types in different seasons as well as the higher and lower values recorded during the study were within the normal range only. Similar findings were reported by Kaneko et al., 2008; Serum levels of ALT in cattle ranged from 11$40 \mathrm{U} / \mathrm{l}$. Moreover, ALT in general has more significance in non-ruminants.

\section{Choline Esterase (ChE)}

Activity of Choline esterase enzyme (ChE) was found to differ significantly $(\mathrm{P}<0.01)$ between seasons in all the zones. There was no significant difference between the housing systems in all the zones in the ChE level. 
Table.1 Zone wise details of Mean \pm SE of activity of AST and ALT enzymes in various housing types during South West monsoon (SWM), North East monsoon (NEM), Winter and Summer seasons

\begin{tabular}{|c|c|c|c|c|c|c|c|c|c|c|c|c|c|c|c|c|c|c|c|c|c|c|}
\hline \multirow[t]{4}{*}{ Name } & \multirow[t]{4}{*}{ Seasons } & \multicolumn{21}{|c|}{ Agro Climatic Zones } \\
\hline & & \multicolumn{7}{|c|}{ Southern } & \multicolumn{7}{|c|}{ Cauvery Delta } & \multicolumn{7}{|c|}{ High Rainfall } \\
\hline & & \multicolumn{7}{|c|}{ Housing Type } & \multicolumn{7}{|c|}{ Housing Type } & \multicolumn{7}{|c|}{ Housing Type } \\
\hline & & Thatched & Tiled & Asbestos & Metal & Open & Total & Anova & Thatched & Tiled & Asbestos & Metal & Open & Total & Anova & Thatched & Tiled & Asbestos & Metal & Open & Total & Anova \\
\hline \multirow[t]{6}{*}{$\begin{array}{c}\text { AST } \\
(\mathrm{U} / \mathrm{L})\end{array}$} & SWM & $\begin{array}{l}81.4 \\
\pm 5.8\end{array}$ & $\begin{array}{l}94.2 \\
\pm 4.0\end{array}$ & $\begin{array}{l}72.2 \\
\pm 3.9\end{array}$ & $\begin{array}{l}83.4 \\
\pm 3.2\end{array}$ & $\begin{array}{r}72.9 \\
\pm 2.2\end{array}$ & $\begin{array}{r}80.9 \\
\pm 2.2\end{array}$ & \multirow{6}{*}{$\begin{array}{c}\mathrm{S}(0.001) \\
\mathrm{HT} \\
(0.077) \\
\mathrm{S} * \mathrm{HT} \\
(0.855)\end{array}$} & $\begin{array}{l}87.3 \\
\pm 6.1\end{array}$ & $\begin{array}{l}95.8 \\
\pm 2.7\end{array}$ & $\begin{array}{l}66.9 \\
\pm 0.4\end{array}$ & $\begin{array}{l}70.2 \\
\pm 1.1\end{array}$ & $\begin{array}{r}83.5 \\
\pm 3.6\end{array}$ & $\begin{array}{l}80.8 \\
\pm 2.5\end{array}$ & \multirow{6}{*}{$\begin{array}{c}\mathrm{S}(0.000) \\
\mathrm{HT} \\
(0.001) \\
\mathrm{S} * \mathrm{HT} \\
(0.140)\end{array}$} & $\begin{array}{l}62.0 \\
\pm 7.3\end{array}$ & $\begin{array}{l}63.1 \\
\pm 2.3\end{array}$ & 62.8 & 69.2 & 71.4 & 65.7 & \multirow{6}{*}{$\begin{array}{c}\mathrm{S} \\
(0.000) \\
\mathrm{HT} \\
(0.022) \\
\mathrm{S} * \mathrm{HT} \\
(0.408)\end{array}$} \\
\hline & & & & & & & & & & & & & & & & & & & & & & \\
\hline & NEM & $\begin{array}{l}76.8 \\
\pm 9.5\end{array}$ & $\begin{array}{l}82.1 \\
\pm 7.0\end{array}$ & $\begin{array}{l}88.1 \\
\pm 9.4\end{array}$ & $\begin{array}{l}91.6 \\
\pm 6.8\end{array}$ & $\begin{array}{l}79.7 \\
\pm 6.5\end{array}$ & $\begin{array}{l}83.6 \\
\pm 3.5\end{array}$ & & $\begin{array}{r}80.5 \\
\pm 5.0\end{array}$ & $\begin{array}{l}97.8 \\
\pm 9.2\end{array}$ & $\begin{array}{l}87.7 \\
\pm 6.7\end{array}$ & $\begin{array}{l}85.2 \\
\pm 6.6\end{array}$ & $\begin{array}{c}101.6 \\
\pm 11 \\
6\end{array}$ & $\begin{array}{l}90.6 \\
\pm 3.7\end{array}$ & & $\begin{array}{l}106.5 \\
\pm 4.6\end{array}$ & $\begin{array}{l}107.5 \\
\pm 3.3\end{array}$ & $\begin{array}{c}104.9 \\
\pm 3.3\end{array}$ & $\begin{array}{c}106.4 \\
\pm 4.8\end{array}$ & $\begin{array}{c}104.9 \\
\pm 2.3\end{array}$ & $\begin{array}{c}106 . \\
0 \\
\pm 1.6\end{array}$ & \\
\hline & Winter & $\begin{array}{c}87.3 \\
\pm 11.9\end{array}$ & $\begin{array}{l}87.0 \\
\pm 7.3\end{array}$ & $\begin{array}{l}87.4 \\
\pm 8.0\end{array}$ & $\begin{array}{c}98.4 \\
\pm 10.9\end{array}$ & $\begin{array}{l}78.6 \\
\pm 9.4\end{array}$ & $\begin{array}{l}87.7 \\
\pm 4.2\end{array}$ & & $\begin{array}{l}64.3 \\
\pm 4.6\end{array}$ & $\begin{array}{l}72.6 \\
\pm 3.3\end{array}$ & $\begin{array}{l}53.3 \\
\pm 4.9\end{array}$ & $\begin{array}{l}72.1 \\
\pm 2.8\end{array}$ & $\begin{array}{l}76.6 \\
\pm 8.2\end{array}$ & $\begin{array}{l}67.8 \\
\pm 2.6\end{array}$ & & $\begin{array}{l}119.4 \\
\pm 21.1\end{array}$ & $\begin{array}{l}98.1 \\
\pm 6.8\end{array}$ & $\begin{array}{l}77.7 \\
\pm 6.6\end{array}$ & $\begin{array}{l}116.2 \\
\pm 13.6\end{array}$ & $\begin{array}{l}113.8 \\
\pm 13.3\end{array}$ & $\begin{array}{c}105 \\
0 \\
\pm 6.3\end{array}$ & \\
\hline & Summer & $\begin{array}{l}70.6 \\
\pm 7.1\end{array}$ & $\begin{array}{l}72.7 \\
\pm 6.7\end{array}$ & $\begin{array}{l}73.2 \\
\pm 2.7\end{array}$ & $\begin{array}{l}72.3 \\
\pm 6.9\end{array}$ & $\begin{array}{l}58.7 \\
\pm 6.1\end{array}$ & $\begin{array}{l}69.5 \\
\pm 2.7\end{array}$ & & $\begin{array}{l}66.0 \\
\pm 4.0\end{array}$ & $\begin{array}{l}66.8 \\
\pm 2.2\end{array}$ & $\begin{array}{l}61.7 \\
\pm 6.4\end{array}$ & $\begin{array}{l}67.9 \\
\pm 7.9\end{array}$ & $\begin{array}{l}65.8 \\
\pm 2.7\end{array}$ & $\begin{array}{c}65.6 \\
2.2\end{array}$ & & $\begin{array}{l}66.3 \\
\pm 4.1\end{array}$ & $\begin{array}{l}59.2 \\
\pm 6.6\end{array}$ & $\begin{array}{l}54.1 \\
\pm 6.0\end{array}$ & $\begin{array}{l}68.2 \\
\pm 6.6\end{array}$ & $\begin{array}{l}76.4 \\
\pm 4.9\end{array}$ & $\begin{array}{l}64.8 \\
\pm 2.8\end{array}$ & \\
\hline & Total & $\begin{array}{l}79.0 \\
\pm 4.3\end{array}$ & $\begin{array}{l}84.0 \\
\pm 3.4\end{array}$ & $\begin{array}{l}80.2 \\
\pm 3.5\end{array}$ & $\begin{array}{l}86.4^{\mathrm{e}} \\
\pm 4.0\end{array}$ & $\begin{array}{l}72.5^{\mathrm{d}} \\
\pm 3.5\end{array}$ & $\begin{array}{l}80.4 \\
\pm 1.7\end{array}$ & & $\begin{array}{l}74.5 \\
\pm 3.1\end{array}$ & $\begin{array}{l}83.2^{c} \\
\pm 3.7\end{array}$ & $\begin{array}{c}67.4^{\text {be }} \\
\pm 3.6\end{array}$ & $\begin{array}{l}73.8 \\
\pm 2.9\end{array}$ & $\begin{array}{l}81.9^{c} \\
\pm 4.4\end{array}$ & $\begin{array}{l}76.2 \\
\pm 1.7\end{array}$ & & $\begin{array}{l}88.5 \\
\pm 7.5\end{array}$ & $\begin{array}{l}82.0 \\
\pm 5.0\end{array}$ & $\begin{array}{l}74.9 \\
\pm 4.7^{\mathrm{e}}\end{array}$ & $\begin{array}{l}90.0 \\
\pm 5.9\end{array}$ & $\begin{array}{c}91.6 \\
\pm 5.4^{\mathrm{c}}\end{array}$ & $\begin{array}{l}85.4 \\
\pm 2.6\end{array}$ & \\
\hline \multirow[t]{5}{*}{$\begin{array}{c}\text { ALT } \\
\text { (U/L) }\end{array}$} & SWM & $\begin{array}{l}28.7 \\
\pm 1.8\end{array}$ & $\begin{array}{l}31.1 \\
\pm 2.1\end{array}$ & $\begin{array}{r}35.3 \\
\pm 3.9\end{array}$ & $\begin{array}{l}24.9 \\
\pm 1.1\end{array}$ & $\begin{array}{l}26.6 \\
\pm 1.4\end{array}$ & $\begin{array}{l}29.3 \\
\pm 1.2\end{array}$ & \multirow{5}{*}{$\begin{array}{c}\mathrm{S}(0.000) \\
\mathrm{HT} \\
(0.000) \\
\mathrm{S} * \mathrm{HT} \\
(0.011)\end{array}$} & $\begin{array}{l}30.1 \\
\pm 1.1\end{array}$ & $\begin{array}{l}30.0 \\
\pm 0.9\end{array}$ & $\begin{array}{l}24.9 \\
\pm 0.4\end{array}$ & $\begin{array}{l}22.1 \\
\pm 0.4\end{array}$ & $\begin{array}{l}24.4 \\
\pm 1.0\end{array}$ & $\begin{array}{l}26.3 \\
\pm 0.7\end{array}$ & \multirow{5}{*}{$\begin{array}{c}\mathrm{S}(0.000) \\
\mathrm{HT} \\
(0.112) \\
\mathrm{S} * \mathrm{HT} \\
(0.149)\end{array}$} & $\begin{array}{l}26.6 \\
\pm 3.6\end{array}$ & $\begin{array}{l}25.5 \\
\pm 1.9\end{array}$ & $\begin{array}{l}22.7 \\
\pm 0.9\end{array}$ & $\begin{array}{l}26.8 \\
\pm 4.2\end{array}$ & $\begin{array}{l}28.5 \\
\pm 4.9\end{array}$ & $\begin{array}{l}26.0 \\
\pm 1.5\end{array}$ & \multirow{5}{*}{$\begin{array}{c}\mathrm{S} \\
(0.000) \\
\mathrm{HT} \\
(0.400) \\
\mathrm{S} * \mathrm{HT} \\
(0.082)\end{array}$} \\
\hline & NEM & $\begin{array}{l}22.3 \\
\pm 2.0\end{array}$ & $\begin{array}{l}25.0 \\
\pm 3.7\end{array}$ & $\begin{array}{l}34.9 \\
\pm 4.0\end{array}$ & $\begin{array}{l}33.2 \\
\pm 2.5\end{array}$ & $\begin{array}{l}27.4 \\
\pm 1.9\end{array}$ & $\begin{array}{l}28.6 \\
\pm 1.5\end{array}$ & & $\begin{array}{l}29.0 \\
\pm 3.4\end{array}$ & $\begin{array}{l}27.7 \\
\pm 1.8\end{array}$ & $\begin{array}{l}28.8 \\
\pm 3.8\end{array}$ & $\begin{array}{l}27.8 \\
\pm 3.3\end{array}$ & $\begin{array}{l}26.1 \\
\pm 2.9\end{array}$ & $\begin{array}{l}27.9 \\
\pm 1.3\end{array}$ & & $\begin{array}{l}28.1 \\
\pm 2.6\end{array}$ & $\begin{array}{l}30.8 \\
\pm 1.5\end{array}$ & $\begin{array}{r}34.6 \\
\pm 3.6\end{array}$ & $\begin{array}{r}25.3 \\
\pm 3.2\end{array}$ & $\begin{array}{l}20.9 \\
\pm 2.5\end{array}$ & $\begin{array}{l}27.9 \\
\pm 1.4\end{array}$ & \\
\hline & Winter & $\begin{array}{l}23.4 \\
\pm 2.0\end{array}$ & $\begin{array}{l}42.8 \\
\pm 5.0\end{array}$ & $\begin{array}{l}41.4 \\
\pm 2.7\end{array}$ & $\begin{array}{l}38.2 \\
\pm 4.6\end{array}$ & $\begin{array}{l}27.3 \\
\pm 3.7\end{array}$ & $\begin{array}{l}34.6 \\
\pm 2.1\end{array}$ & & $\begin{array}{l}20.5 \\
\pm 2.0\end{array}$ & $\begin{array}{l}22.9 \\
\pm 3.0\end{array}$ & $\begin{array}{l}14.6 \\
\pm 2.1\end{array}$ & $\begin{array}{l}19.4 \\
\pm 1.5\end{array}$ & $\begin{array}{l}24.1 \\
\pm 1.3\end{array}$ & $\begin{array}{l}20.3 \\
\pm 1.1\end{array}$ & & $\begin{array}{l}34.1 \\
\pm 4.5\end{array}$ & $\begin{array}{l}39.5 \\
\pm 3.7\end{array}$ & $\begin{array}{l}25.6 \\
\pm 3.6\end{array}$ & $\begin{array}{l}35.5 \\
\pm 4.6\end{array}$ & $\begin{array}{l}36.4 \\
\pm 5.0\end{array}$ & $\begin{array}{l}34.2 \\
\pm 2.0\end{array}$ & \\
\hline & Summer & $\begin{array}{l}28.2 \\
\pm 2.2\end{array}$ & $\begin{array}{l}23.6 \\
\pm 3.3\end{array}$ & $\begin{array}{l}28.4 \\
\pm 5.3\end{array}$ & $\begin{array}{l}26.2 \\
\pm 1.9\end{array}$ & $\begin{array}{l}19.3 \\
\pm 3.0\end{array}$ & $\begin{array}{l}25.1 \\
\pm 1.5\end{array}$ & & $\begin{array}{r}18.9 \\
\pm 1.9\end{array}$ & $\begin{array}{l}18.6 \\
\pm 0.8\end{array}$ & $\begin{array}{r}18.3 \\
\pm 1.6\end{array}$ & $\begin{array}{l}18.5 \\
\pm 3.1\end{array}$ & $\begin{array}{l}22.0 \\
\pm 2.3\end{array}$ & $\begin{array}{l}19.2 \\
\pm 0.9\end{array}$ & & $\begin{array}{r}18.0 \\
\pm 3.1\end{array}$ & $\begin{array}{l}21.7 \\
\pm 3.0\end{array}$ & $\begin{array}{l}16.0 \\
\pm 2.2\end{array}$ & $\begin{array}{l}22.1 \\
\pm 1.2\end{array}$ & $\begin{array}{l}21.0 \\
\pm 1.4\end{array}$ & $\begin{array}{r}19.8 \\
\pm 1.1\end{array}$ & \\
\hline & Total & $\begin{array}{l}25.6^{\mathrm{c}} \\
\pm 1.1\end{array}$ & $\begin{array}{l}30.6 \\
\pm 2.3\end{array}$ & $\begin{array}{c}35.0^{\mathrm{a}, \mathrm{e}} \\
\pm 2.1\end{array}$ & $\begin{array}{l}30.6 \\
\pm 1.7\end{array}$ & $\begin{array}{l}25.1 \\
\pm 1.4\end{array}$ & $\begin{array}{l}29.4 \\
\pm 0.9\end{array}$ & & $\begin{array}{l}24.6 \\
\pm 1.5\end{array}$ & $\begin{array}{l}24.8 \\
\pm 1.3\end{array}$ & $\begin{array}{l}21.6 \\
\pm 1.6\end{array}$ & $\begin{array}{l}21.9 \\
\pm 1.4\end{array}$ & $\begin{array}{l}24.1 \\
\pm 1.0\end{array}$ & $\begin{array}{l}23.4 \\
\pm 0.6\end{array}$ & & $\begin{array}{l}26.7 \\
\pm 2.0\end{array}$ & $\begin{array}{l}29.4 \\
\pm 1.9\end{array}$ & $\begin{array}{r}24.7 \\
\pm 1.9\end{array}$ & $\begin{array}{l}27.4 \\
\pm 1.9\end{array}$ & $\begin{array}{l}26.7 \\
\pm 2.2\end{array}$ & $\begin{array}{l}27.0 \\
\pm 0.9\end{array}$ & \\
\hline
\end{tabular}

S. Season, HT - Housing type, S*HT - Season verses Housing interaction. Figures in parenthesis indicates significance 
Table.2 Zone wise details of Mean \pm SE of activity of ChE and LDH enzymes in various housing types during South West monsoon (SWM), North East monsoon (NEM), Winter and Summer seasons

\begin{tabular}{|c|c|c|c|c|c|c|c|c|c|c|c|c|c|c|c|c|c|c|c|c|c|c|}
\hline \multirow[t]{4}{*}{ Name } & \multirow[t]{4}{*}{ Seasons } & \multicolumn{21}{|c|}{ Agro Climatic Zones } \\
\hline & & \multicolumn{7}{|c|}{ Southern } & \multicolumn{7}{|c|}{ Cauvery Delta } & \multicolumn{7}{|c|}{ High Rainfall } \\
\hline & & \multicolumn{7}{|c|}{ Housing Type } & \multicolumn{7}{|c|}{ Housing Type } & \multicolumn{7}{|c|}{ Housing Type } \\
\hline & & Thatched & Tiled & Asbestos & Metal & Open & Total & Anova & Thatched & Tiled & Asbestos & Metal & Open & Total & Anova & Thatched & Tiled & Asbestos & Metal & Open & Total & Anova \\
\hline \multirow[t]{5}{*}{$\begin{array}{c}\mathrm{ChE} \\
(\mathrm{U} / \mathrm{L})\end{array}$} & SWM & $\begin{array}{l}126.4 \\
\pm 12.1\end{array}$ & $\begin{array}{c}120.7 \\
\pm 0.9\end{array}$ & $\begin{array}{l}115.8 \\
\pm 10.9\end{array}$ & $\begin{array}{l}156.5 \\
\pm 19.9\end{array}$ & $\begin{array}{l}165.9 \\
\pm 8.0\end{array}$ & $\begin{array}{c}137.0 \\
\pm 6.3\end{array}$ & \multirow{5}{*}{$\begin{array}{c}\mathrm{S}(0.000) \\
\mathrm{HT} \\
(0.380) \\
\mathrm{S} * \mathrm{HT} \\
(0.159)\end{array}$} & $\begin{array}{r}104.3 \\
\pm 11.6\end{array}$ & $\begin{array}{c}100.2 \\
\pm 4.3\end{array}$ & $\begin{array}{l}79.5 \\
\pm 0.3\end{array}$ & $\begin{array}{l}86.2 \\
\pm 1.8\end{array}$ & $\begin{array}{c}91.1 \\
\pm 10.7\end{array}$ & $\begin{array}{l}92.3 \\
\pm 3.5\end{array}$ & \multirow{5}{*}{$\begin{array}{c}\mathrm{S}(0.000) \\
\mathrm{HT} \\
(0.149) \\
\mathrm{S} * \mathrm{HT} \\
(0.708)\end{array}$} & $\begin{array}{c}106.8 \\
\pm 9.8\end{array}$ & $\begin{array}{l}131.7 \\
\pm 9.7\end{array}$ & $\begin{array}{l}136.7 \\
\pm 10.1\end{array}$ & $\begin{array}{l}115.6 \\
\pm 8.4\end{array}$ & $\begin{array}{l}110.2 \\
\pm 19.6\end{array}$ & $\begin{array}{c}120.2 \\
\pm 5.5\end{array}$ & \multirow{5}{*}{$\begin{array}{c}\mathrm{S} \\
(.000) \\
\mathrm{HT} \\
(0.322) \\
\mathrm{S} * \mathrm{HT} \\
(0.232)\end{array}$} \\
\hline & NEM & $\begin{array}{l}119.2 \\
\pm 20.9\end{array}$ & $\begin{array}{l}180.9 \\
\pm 60.9\end{array}$ & $\begin{array}{l}205.0 \\
\pm 95.9\end{array}$ & $\begin{array}{l}233.7 \\
\pm 82.4\end{array}$ & $\begin{array}{l}153.2 \\
\pm 37.4\end{array}$ & $\begin{array}{l}178.4 \\
\pm 28.2\end{array}$ & & $\begin{array}{l}282.7 \\
\pm 48.5\end{array}$ & $\begin{array}{l}176.8 \\
\pm 47.2\end{array}$ & $\begin{array}{l}154.6 \\
\pm 33.3\end{array}$ & $\begin{array}{l}150.9 \\
\pm 32.4\end{array}$ & $\begin{array}{l}108.5 \\
\pm 19.5\end{array}$ & $\begin{array}{l}174.7 \\
\pm 19.1\end{array}$ & & $\begin{array}{l}60.9 \\
\pm 5.1\end{array}$ & $\begin{array}{c}60.8 \\
\pm 10.3\end{array}$ & $\begin{array}{c}98.5 \\
\pm 23.4\end{array}$ & $\begin{array}{l}115.8 \\
\pm 22.8\end{array}$ & $\begin{array}{c}56.9 \\
\pm 16.3\end{array}$ & $\begin{array}{l}78.6 \\
\pm 8.4\end{array}$ & \\
\hline & Winter & $\begin{array}{c}493.9 \\
\pm 140.4\end{array}$ & $\begin{array}{l}290.4 \\
\pm 30.4\end{array}$ & $\begin{array}{l}210.4 \\
\pm 21.1\end{array}$ & $\begin{array}{l}236.4 \\
\pm 26.0\end{array}$ & $\begin{array}{c}421.2 \\
\pm 169.9\end{array}$ & $\begin{array}{l}330.5 \\
\pm 46.5\end{array}$ & & $\begin{array}{r}351.3 \\
\pm 59.1\end{array}$ & $\begin{array}{c}344.4 \\
\pm 116.4\end{array}$ & $\begin{array}{l}250.5 \\
\pm 21.4\end{array}$ & $\begin{array}{l}233.4 \\
\pm 17.7\end{array}$ & $\begin{array}{l}270.6 \\
\pm 32.1\end{array}$ & $\begin{array}{l}290.0 \\
\pm 27.0\end{array}$ & & $\begin{array}{l}204.8 \\
\pm 27.4\end{array}$ & $\begin{array}{l}252.2 \\
\pm 29.3\end{array}$ & $\begin{array}{l}213.6 \\
\pm 29.1\end{array}$ & $\begin{array}{l}219.6 \\
\pm 19.8\end{array}$ & $\begin{array}{l}249.8 \\
\pm 15.9\end{array}$ & $\begin{array}{l}228.0 \\
\pm 11.0\end{array}$ & \\
\hline & Summer & $\begin{array}{c}643.2 \\
\pm 100.4\end{array}$ & $\begin{array}{l}487.5 \\
\pm 63.9\end{array}$ & $\begin{array}{l}474.3 \\
\pm 66.0\end{array}$ & $\begin{array}{c}670.7 \\
\pm 130.8\end{array}$ & $\begin{array}{l}405.7 \\
\pm 72.0\end{array}$ & $\begin{array}{l}536.3 \\
\pm 42.1\end{array}$ & & $\begin{array}{l}392.0 \\
\pm 70.1\end{array}$ & $\begin{array}{l}429.2 \\
\pm 30.5\end{array}$ & $\begin{array}{l}469.0 \\
\pm 79.3\end{array}$ & $\begin{array}{l}379.7 \\
\pm 61.4\end{array}$ & $\begin{array}{l}375.0 \\
\pm 53.7\end{array}$ & $\begin{array}{l}409.0 \\
\pm 26.3\end{array}$ & & $\begin{array}{l}484.2 \\
\pm 65.5\end{array}$ & $\begin{array}{l}477.8 \\
\pm 63.2\end{array}$ & $\begin{array}{c}607.2 \\
\pm 124.8\end{array}$ & $\begin{array}{l}357.8 \\
\pm 28.6\end{array}$ & $\begin{array}{l}507.7 \\
\pm 80.8\end{array}$ & $\begin{array}{l}486.9 \\
\pm 36.0\end{array}$ & \\
\hline & Total & $\begin{array}{l}345.7 \\
\pm 62.7\end{array}$ & $\begin{array}{r}269.9 \\
\pm 36.3\end{array}$ & $\begin{array}{l}251.4 \\
\pm 39.4\end{array}$ & $\begin{array}{l}324.3 \\
\pm 56.0\end{array}$ & $\begin{array}{l}286.5 \\
\pm 51.3\end{array}$ & $\begin{array}{l}295.5 \\
\pm 22.3\end{array}$ & & $\begin{array}{r}282.6 \\
\pm 33.5\end{array}$ & $\begin{array}{l}262.6 \\
\pm 40.6\end{array}$ & $\begin{array}{l}238.4 \\
\pm 36.8\end{array}$ & $\begin{array}{l}212.5 \\
\pm 28.3\end{array}$ & $\begin{array}{l}211.3 \\
\pm 29.0\end{array}$ & $\begin{array}{l}241.5 \\
\pm 15.2\end{array}$ & & $\begin{array}{l}214.2 \\
\pm 38.1\end{array}$ & $\begin{array}{l}230.6 \\
\pm 36.9\end{array}$ & $\begin{array}{l}264.0 \\
\pm 52.1\end{array}$ & $\begin{array}{l}202.2 \\
\pm 23.0\end{array}$ & $\begin{array}{l}231.1 \\
\pm 41.6\end{array}$ & $\begin{array}{l}228.4 \\
\pm 17.4\end{array}$ & \\
\hline \multirow[t]{5}{*}{$\begin{array}{l}\text { LDH } \\
(\mathrm{U} / \mathrm{L})\end{array}$} & SWM & $\begin{array}{l}1482.9 \\
\pm 143.7\end{array}$ & $\begin{array}{r}1228.5 \\
\pm 122.7\end{array}$ & $\begin{array}{l}1432.2 \\
\pm 76.3\end{array}$ & $\begin{array}{c}1869.0 \\
\pm 90.4\end{array}$ & $\begin{array}{c}1143.1 \\
\pm 47.1\end{array}$ & $\begin{array}{l}1431.1 \\
\pm 63.1\end{array}$ & \multirow[t]{5}{*}{$\begin{array}{c}\mathrm{S} \\
(0.000) \\
\mathrm{HT} \\
(0.000) \\
\mathrm{S} * \mathrm{HT} \\
(0.000)\end{array}$} & $\begin{array}{c}1388.6 \\
\pm 73.6\end{array}$ & $\begin{array}{l}1420.5 \\
\pm 24.4\end{array}$ & $\begin{array}{l}1021.4 \\
\pm 189.3\end{array}$ & $\begin{array}{c}1821.9 \\
\pm 49.2\end{array}$ & $\begin{array}{c}1493.4 \\
\pm 78.7\end{array}$ & $\begin{array}{c}1429.2 \\
\pm 63.2\end{array}$ & \multirow[t]{5}{*}{$\begin{array}{c}\mathrm{S} \\
(0.000) \\
\mathrm{HT} \\
(0.004) \\
\mathrm{S} * \mathrm{HT} \\
(0.000)\end{array}$} & $\begin{array}{c}1128.9 \\
\pm 21.3\end{array}$ & $\begin{array}{l}1210.8 \\
\pm 65.2\end{array}$ & $\begin{array}{c}1188.1 \\
\pm 45.0\end{array}$ & $\begin{array}{l}1211.8 \\
\pm 109.1\end{array}$ & $\begin{array}{c}1247.2 \\
\pm 53.2\end{array}$ & $\begin{array}{c}1197.3 \\
\pm 28.2\end{array}$ & \multirow[t]{5}{*}{$\begin{array}{c}\mathrm{S} \\
(0.00) \\
\mathrm{HT} \\
(.849) \\
\mathrm{S} * \mathrm{HT} \\
(.094)\end{array}$} \\
\hline & NEM & $\begin{array}{l}1525.6 \\
\pm 107.8\end{array}$ & $\begin{array}{r}1875.7 \\
\pm 163.8\end{array}$ & $\begin{array}{r}1683.1 \\
\pm 205.6\end{array}$ & $\begin{array}{l}2169.7 \\
\pm 123.0\end{array}$ & $\begin{array}{l}1702.4 \\
\pm 127.3\end{array}$ & $\begin{array}{c}1791.3 \\
\pm 74.3\end{array}$ & & $\begin{array}{l}1604.7 \\
\pm 99.5\end{array}$ & $\begin{array}{r}1784.1 \\
\pm 127.9\end{array}$ & $\begin{array}{l}1609.5 \\
\pm 105.3\end{array}$ & $\begin{array}{l}1597.8 \\
\pm 104.6\end{array}$ & $\begin{array}{l}1872.8 \\
\pm 105.3\end{array}$ & $\begin{array}{l}1693.8 \\
\pm 49.9\end{array}$ & & $\begin{array}{c}2123.1 \\
\pm 98.4\end{array}$ & $\begin{array}{l}1957.6 \\
\pm 69.8\end{array}$ & $\begin{array}{l}2330.4 \\
\pm 126.9\end{array}$ & $\begin{array}{c}2050.7 \\
\pm 92.7\end{array}$ & $\begin{array}{c}2025.8 \\
\pm 91.4\end{array}$ & $\begin{array}{c}2097.5 \\
\pm 47.0\end{array}$ & \\
\hline & Winter & $\begin{array}{l}1099.1 \\
\pm 237.2\end{array}$ & $\begin{array}{l}2391.4 \\
\pm 116.5\end{array}$ & $\begin{array}{r}1999.6 \\
\pm 142.9\end{array}$ & $\begin{array}{l}2020.4 \\
\pm 109.7\end{array}$ & $\begin{array}{r}1509.1 \\
\pm 333.7\end{array}$ & $\begin{array}{l}1803.9 \\
\pm 119.8\end{array}$ & & $\begin{array}{r}737.5 \\
\pm 69.6\end{array}$ & $\begin{array}{l}678.4 \\
\pm 14.7\end{array}$ & $\begin{array}{l}593.0 \\
\pm 40.9\end{array}$ & $\begin{array}{l}652.1 \\
\pm 61.0\end{array}$ & $\begin{array}{c}841.1 \\
\pm 150.3\end{array}$ & $\begin{array}{r}700.4 \\
\pm 37.2\end{array}$ & & $\begin{array}{l}2179.8 \\
\pm 249.8\end{array}$ & $\begin{array}{l}2103.1 \\
\pm 93.2\end{array}$ & $\begin{array}{l}1679.4 \\
\pm 139.6\end{array}$ & $\begin{array}{l}2208.2 \\
\pm 274.6\end{array}$ & $\begin{array}{l}2052.3 \\
\pm 64.2\end{array}$ & $\begin{array}{l}2044.5 \\
\pm 84.4\end{array}$ & \\
\hline & Summer & $\begin{array}{l}807.0 \\
\pm 66.7\end{array}$ & $\begin{array}{l}832.5 \\
\pm 72.5\end{array}$ & $\begin{array}{l}728.0 \\
\pm 47.8\end{array}$ & $\begin{array}{l}821.3 \\
\pm 60.5\end{array}$ & $\begin{array}{l}831.5 \\
\pm 51.6\end{array}$ & $\begin{array}{l}804.1 \\
\pm 26.2\end{array}$ & & $\begin{array}{r}767.5 \\
\pm 33.0\end{array}$ & $\begin{array}{l}685.8 \\
\pm 47.6\end{array}$ & $\begin{array}{l}682.3 \\
\pm 50.3\end{array}$ & $\begin{array}{l}520.7 \\
\pm 89.8\end{array}$ & $\begin{array}{l}701.7 \\
\pm 58.7\end{array}$ & $\begin{array}{l}671.6 \\
\pm 28.8\end{array}$ & & $\begin{array}{l}717.5 \\
\pm 72.4\end{array}$ & $\begin{array}{c}687.8 \\
\pm 130.4\end{array}$ & $\begin{array}{r}636.3 \\
\pm 53.1\end{array}$ & $\begin{array}{l}667.7 \\
\pm 29.9\end{array}$ & $\begin{array}{l}769.8 \\
\pm 58.3\end{array}$ & $\begin{array}{l}695.8 \\
\pm 32.9\end{array}$ & \\
\hline & Total & $\begin{array}{c}1228.6^{\mathrm{b}, \mathrm{d}} \\
\pm 94.0\end{array}$ & $\begin{array}{c}1582.0 \\
\mathrm{a}, \mathrm{e} \\
\pm 137.2\end{array}$ & $\begin{array}{r}1460.7 \\
\pm 115.7\end{array}$ & $\begin{array}{c}1720.1 \\
\mathrm{a}, \mathrm{e} \\
\pm 119.7\end{array}$ & $\begin{array}{l}1296.5 \\
\mathrm{~b}, \mathrm{~d} \\
\pm 109.9\end{array}$ & $\begin{array}{c}1457.6 \pm 5 \\
3.7\end{array}$ & & $\begin{array}{c}1124.6 \\
\pm 86.2\end{array}$ & $\begin{array}{l}1142.2 \\
\pm 104.8\end{array}$ & $\begin{array}{l}976.6^{\mathrm{e}} \\
\pm 98.5\end{array}$ & $\begin{array}{l}1148.1 \\
\pm 124.3\end{array}$ & $\begin{array}{l}1227.2^{\mathrm{c}} \\
\pm 110.8\end{array}$ & $\begin{array}{c}1123.7 \\
\pm 47.1\end{array}$ & & $\begin{array}{l}1537.3 \\
\pm 146.8\end{array}$ & $\begin{array}{l}1489.8 \\
\pm 127.3\end{array}$ & $\begin{array}{l}1458.5 \\
\pm 138.3\end{array}$ & $\begin{array}{l}1534.6 \\
\pm 149.6\end{array}$ & $\begin{array}{l}1523.7 \\
\pm 117.5\end{array}$ & $\begin{array}{c}1508.8 \\
\pm 60.0\end{array}$ & \\
\hline
\end{tabular}

S. Season, HT - Housing type, $\mathrm{S} * \mathrm{HT}$ - Season verses Housing interaction. Figures in parenthesis indicates significance 
Table.3 Zone wise details of Mean \pm SE concentration of Cortisol, $\mathrm{T}_{3}$ and $\mathrm{T}_{4}$ hormones in various housing types during South West monsoon (SWM), North East monsoon (NEM), Winter and Summer seasons

\begin{tabular}{|c|c|c|c|c|c|c|c|c|c|c|c|c|c|c|c|c|c|c|c|c|c|c|}
\hline \multirow[t]{4}{*}{ Name } & \multirow[t]{4}{*}{ Seasons } & \multicolumn{21}{|c|}{ Agro Climatic Zones } \\
\hline & & \multirow{2}{*}{\multicolumn{7}{|c|}{$\begin{array}{c}\text { Southern } \\
\text { Housing Type }\end{array}$}} & \multirow{2}{*}{\multicolumn{7}{|c|}{$\begin{array}{l}\text { Cauvery Delta } \\
\text { Housing Type }\end{array}$}} & \multirow{2}{*}{\multicolumn{7}{|c|}{$\begin{array}{l}\text { High Rainfall } \\
\text { Housing Type }\end{array}$}} \\
\hline & & & & & & & & & & & & & & & & & & & & & & \\
\hline & & Thatched & Tiled & Asbestos & Metal & Open & Total & Anova & Thatched & Tiled & Asbestos & Metal & Open & Total & Anova & Thatched & Tiled & Asbestos & Metal & Open & Total & Anova \\
\hline \multirow{6}{*}{$\begin{array}{l}\text { Cortisol } \\
(\mu \mathrm{gg} / \mathrm{dl})\end{array}$} & SWM & $\begin{array}{l}2.285 \\
+0.49\end{array}$ & $\begin{array}{l}2.119 \\
\pm 0.38\end{array}$ & $\begin{array}{l}6.318 \\
+1.88\end{array}$ & $\begin{array}{r}4.221 \\
+1.06\end{array}$ & $\begin{array}{l}5.334 \\
+2.05\end{array}$ & $\begin{array}{l}4.055 \\
+0.64\end{array}$ & \multirow{6}{*}{$\begin{array}{c}\text { S }(0.000) \\
\text { HT }(0.035) \\
\text { S*HT } \\
(0.784)\end{array}$} & $\begin{array}{l}3.844 \\
+0.53\end{array}$ & $\begin{array}{r}7.803 \\
+2.35\end{array}$ & $\begin{array}{r}8.376 \\
+3.02\end{array}$ & $\begin{array}{c}10.500 \\
+2.29\end{array}$ & $\begin{array}{r}8.993 \\
+3.69\end{array}$ & $\begin{array}{r}7.903 \\
+1.15\end{array}$ & \multirow{6}{*}{$\begin{array}{c}\text { S }(0.000) \\
\text { HT }(0.054) \\
\text { S*HT } \\
(0.114)\end{array}$} & $\begin{array}{r}3.210 \\
+1.01\end{array}$ & $\begin{array}{l}2.170 \\
+0.95\end{array}$ & $\begin{array}{r}6.342 \\
+1.32\end{array}$ & $\begin{array}{r}6.113 \\
+2.49\end{array}$ & $\begin{array}{r}6.582 \\
+3.24\end{array}$ & $\begin{array}{l}4.883 \\
+0.90\end{array}$ & \multirow{6}{*}{$\begin{array}{l}\text { S (.000) } \\
\text { HT (.253) } \\
\text { S*HT } \\
(.376)\end{array}$} \\
\hline & & & & & & & & & & & & & & & & & 1 & & & & & \\
\hline & NEM & $\begin{array}{l}3.861 \\
\pm 1.31\end{array}$ & $\begin{array}{l}6.883 \\
\pm 2.10\end{array}$ & $\begin{array}{l}10.061 \\
\pm 2.63\end{array}$ & $\begin{array}{l}12.594 \\
\pm 4.90\end{array}$ & $\begin{array}{l}13.795 \\
\pm 4.22\end{array}$ & $\begin{array}{l}9.439 \\
\pm 1.53\end{array}$ & & $\begin{array}{l}5.956 \\
\pm 0.40\end{array}$ & $\begin{array}{l}10.581 \\
\pm 2.13\end{array}$ & $\begin{array}{l}12.308 \\
\pm 6.37\end{array}$ & $\begin{array}{l}5.910 \\
\pm 2.00\end{array}$ & $\begin{array}{c}17.123 \pm \\
4.08\end{array}$ & $\begin{array}{c}10.37 \\
6 \\
\pm 1.70\end{array}$ & & $\begin{array}{l}8.006 \\
\pm 2.88\end{array}$ & $\begin{array}{l}8.772 \\
\pm 2.35\end{array}$ & $\begin{array}{l}7.177 \\
\pm 2.80\end{array}$ & $\begin{array}{c}10.49 \\
8 \\
\pm 3.32\end{array}$ & $\begin{array}{l}12.69 \\
2 \\
\pm 4.59\end{array}$ & $\begin{array}{l}9.429 \\
\pm 1.41\end{array}$ & \\
\hline & Winter & $\begin{array}{l}12.280 \\
\pm 2.46\end{array}$ & $\begin{array}{l}10.110 \\
\pm 5.11\end{array}$ & $\begin{array}{l}8.399 \\
\pm 2.97\end{array}$ & $\begin{array}{l}16.054 \\
\pm 6.79\end{array}$ & $\begin{array}{l}15.781 \\
\pm 2.80\end{array}$ & $\begin{array}{c}12.52 \\
5 \\
\pm 1.89\end{array}$ & & $\begin{array}{l}8.648 \\
\pm 1.87\end{array}$ & $\begin{array}{l}9.076 \\
\pm 2.01\end{array}$ & $\begin{array}{l}11.267 \\
\pm 3.37\end{array}$ & $\begin{array}{l}27.954 \\
\pm 12.36\end{array}$ & $\begin{array}{l}21.146 \\
\pm 3.45\end{array}$ & $\begin{array}{c}15.61 \\
8 \\
\pm 2.89\end{array}$ & & $\begin{array}{l}32.781 \\
\pm 12.09\end{array}$ & $\begin{array}{c}29.20 \\
3 \\
\pm 10.1 \\
6\end{array}$ & $\begin{array}{l}17.617 \\
\pm 2.48\end{array}$ & $\begin{array}{c}35.30 \\
6 \\
\pm 6.04\end{array}$ & $\begin{array}{l}18.41 \\
5 \\
\pm 3.60\end{array}$ & $\begin{array}{c}26.66 \\
5 \\
\pm 3.51\end{array}$ & \\
\hline & Summer & $\begin{array}{l}3.811 \\
\pm 0.73\end{array}$ & $\begin{array}{l}1.895 \\
\pm 0.33\end{array}$ & $\begin{array}{l}3.558 \\
\pm 0.91\end{array}$ & $\begin{array}{l}2.524 \\
\pm 0.49\end{array}$ & $\begin{array}{l}8.786 \\
\pm 2.33\end{array}$ & $\begin{array}{l}4.115 \\
\pm 0.67\end{array}$ & & $\begin{array}{l}4.116 \\
\pm 1.56\end{array}$ & $\begin{array}{l}2.474 \\
\pm 0.33\end{array}$ & $\begin{array}{l}2.738 \\
\pm 0.56\end{array}$ & $\begin{array}{l}2.674 \\
\pm 0.29\end{array}$ & $\begin{array}{l}3.545 \\
\pm 0.77\end{array}$ & $\begin{array}{l}3.109 \\
\pm 0.36\end{array}$ & & $\begin{array}{l}13.554 \\
\pm 1.13\end{array}$ & $\begin{array}{l}5.286 \\
\pm 2.24\end{array}$ & $\begin{array}{l}3.013 \\
\pm 0.79\end{array}$ & $\begin{array}{l}7.140 \\
\pm 2.49\end{array}$ & $\begin{array}{l}5.827 \\
\pm 1.97\end{array}$ & $\begin{array}{l}6.964 \\
\pm 1.01\end{array}$ & \\
\hline & Total & $\begin{array}{l}5.559 \\
\pm 1.06\end{array}$ & $\begin{array}{l}5.251^{\mathrm{e}} \\
\pm 1.48\end{array}$ & $\begin{array}{l}7.084 \\
\pm 1.16\end{array}$ & $\begin{array}{l}8.848 \\
\pm 2.29\end{array}$ & $\begin{array}{c}10.924^{\mathrm{B}} \\
\pm 1.63\end{array}$ & $\begin{array}{l}7.533 \\
\pm 0.72\end{array}$ & & $\begin{array}{l}5.641 \\
\pm 0.71\end{array}$ & $\begin{array}{l}7.483 \\
\pm 1.08\end{array}$ & $\begin{array}{l}8.673 \\
\pm 1.98\end{array}$ & $\begin{array}{l}11.760 \\
\pm 3.60\end{array}$ & $\begin{array}{l}12.702 \\
\pm 2.09\end{array}$ & $\begin{array}{l}9.252 \\
\pm 0.97\end{array}$ & & $\begin{array}{l}14.388 \\
\pm 3.74\end{array}$ & $\begin{array}{l}11.35 \\
8 \\
\pm 3.33\end{array}$ & $\begin{array}{l}8.537 \\
\pm 1.48\end{array}$ & $\begin{array}{c}14.76 \\
4 \\
\pm 3.08\end{array}$ & $\begin{array}{l}10.87 \\
9 \\
\pm 1.94\end{array}$ & $\begin{array}{c}11.98 \\
5 \\
\pm 1.27\end{array}$ & \\
\hline \multirow[t]{5}{*}{$\begin{array}{c}\mathrm{T}_{3} \\
(\mathrm{ng} / \mathrm{dl})\end{array}$} & SWM & $\begin{array}{c}3.34 \\
\pm 0.62\end{array}$ & $\begin{array}{c}3.41 \\
\pm 0.68\end{array}$ & $\begin{array}{r}3.78 \\
\pm 0.77\end{array}$ & $\begin{array}{c}1.92 \\
\pm 0.23\end{array}$ & $\begin{array}{r}3.40 \\
\pm 1.27\end{array}$ & $\begin{array}{l}3.17 \\
\pm 0.35\end{array}$ & \multirow{5}{*}{$\begin{array}{c}\text { S }(0.000) \\
\text { HT }(0.391) \\
\text { S*HT } \\
(0.185)\end{array}$} & $\begin{array}{l}3.47 \\
\pm 0.78\end{array}$ & $\begin{array}{r}5.30 \\
\pm 1.72\end{array}$ & $\begin{array}{r}3.69 \\
\pm 0.81\end{array}$ & $\begin{array}{c}5.07 \\
\pm 0.27\end{array}$ & $\begin{array}{r}4.79 \\
\pm 0.92\end{array}$ & $\begin{array}{r}4.46 \\
\pm 0.44\end{array}$ & \multirow{5}{*}{$\begin{array}{c}\mathrm{S}(0.000) \\
\mathrm{HT}(0.152) \\
\text { S*HT } \\
(0.602)\end{array}$} & $\begin{array}{c}2.55 \\
\pm 0.76\end{array}$ & $\begin{array}{c}3.41 \\
\pm 0.69\end{array}$ & $\begin{array}{c}5.05 \\
\pm 0.81\end{array}$ & $\begin{array}{r}6.27 \\
\pm 1.37\end{array}$ & $\begin{array}{c}4.36 \\
\pm 1.04\end{array}$ & $\begin{array}{l}4.332 \\
\pm 0.46\end{array}$ & \multirow{5}{*}{$\begin{array}{c}\mathrm{S}(0.000) \\
\mathrm{HT}(0.390) \\
\mathrm{S} * \mathrm{HT} \\
(0.062)\end{array}$} \\
\hline & NEM & $\begin{array}{r}3.48 \\
\pm 1.45\end{array}$ & $\begin{array}{c}1.51 \\
\pm 0.31\end{array}$ & $\begin{array}{r}1.46 \\
\pm 0.19\end{array}$ & $\begin{array}{l}2.26 \\
\pm 0.67\end{array}$ & $\begin{array}{l}2.26 \\
\pm 0.41\end{array}$ & $\begin{array}{r}2.19 \\
\pm 0.34\end{array}$ & & $\begin{array}{r}3.56 \\
\pm 0.74\end{array}$ & $\begin{array}{l}2.14 \\
\pm 0.44\end{array}$ & $\begin{array}{r}1.82 \\
\pm 0.30\end{array}$ & $\begin{array}{r}3.80 \\
\pm 1.52\end{array}$ & $\begin{array}{r}3.20 \\
\pm 0.77\end{array}$ & $\begin{array}{r}2.90 \\
\pm 0.38\end{array}$ & & $\begin{array}{r}6.92 \\
\pm 2.38\end{array}$ & $\begin{array}{c}1.93 \\
\pm 0.54\end{array}$ & $\begin{array}{r}3.80 \\
\pm 0.71\end{array}$ & $\begin{array}{c}3.35 \\
\pm 0.74\end{array}$ & $\begin{array}{r}2.53 \\
\pm 0.57\end{array}$ & $\begin{array}{c}3.71 \\
\pm 0.59\end{array}$ & \\
\hline & Winter & $\begin{array}{r}2.06 \\
\pm 0.76\end{array}$ & $\begin{array}{l}2.67 \\
\pm 0.55\end{array}$ & $\begin{array}{r}1.18 \\
\pm 0.22\end{array}$ & $\begin{array}{r}2.35 \\
\pm 0.57\end{array}$ & $\begin{array}{l}3.15 \\
\pm 0.43\end{array}$ & $\begin{array}{r}2.28 \\
\pm 0.25\end{array}$ & & $\begin{array}{c}1.32 \\
\pm 0.27\end{array}$ & $\begin{array}{c}3.58 \\
\pm 1.91\end{array}$ & $\begin{array}{c}2.18 \\
\pm 0.37\end{array}$ & $\begin{array}{r}5.66 \\
\pm 2.58\end{array}$ & $\begin{array}{r}3.77 \\
\pm 1.04\end{array}$ & $\begin{array}{c}3.30 \\
\pm 0.69\end{array}$ & & $\begin{array}{c}4.84 \\
\pm 2.21\end{array}$ & $\begin{array}{c}3.12 \\
\pm 0.47\end{array}$ & $\begin{array}{l}4.94 \\
\pm 0.87\end{array}$ & $\begin{array}{c}3.68 \\
\pm 1.57\end{array}$ & $\begin{array}{c}6.74 \\
\pm 2.18\end{array}$ & $\begin{array}{l}4.66 \\
\pm 0.71\end{array}$ & \\
\hline & Summer & $\begin{array}{c}1.05 \\
\pm 0.05\end{array}$ & $\begin{array}{c}1.91 \\
\pm 0.18\end{array}$ & $\begin{array}{c}1.05 \\
\pm 0.13\end{array}$ & $\begin{array}{c}0.78 \\
\pm 0.12\end{array}$ & $\begin{array}{c}0.73 \\
\pm 0.03\end{array}$ & $\begin{array}{c}1.10 \\
\pm 0.09\end{array}$ & & $\begin{array}{c}0.90 \\
\pm 0.17\end{array}$ & $\begin{array}{c}1.16 \\
\pm 0.02\end{array}$ & $\begin{array}{r}1.40 \\
\pm 0.08\end{array}$ & $\begin{array}{r}0.66 \\
\pm 0.05\end{array}$ & $\begin{array}{c}1.73 \\
\pm 0.04\end{array}$ & $\begin{array}{c}1.17 \\
\pm 0.07\end{array}$ & & $\begin{array}{c}1.96 \\
\pm 0.12\end{array}$ & $\begin{array}{c}1.84 \\
\pm 0.24\end{array}$ & $\begin{array}{c}1.38 \\
\pm 0.15\end{array}$ & $\begin{array}{c}0.61 \\
\pm 0.10\end{array}$ & $\begin{array}{c}1.28 \\
\pm 0.09\end{array}$ & $\begin{array}{c}1.41 \\
\pm 0.10\end{array}$ & \\
\hline & Total & $\begin{array}{r}2.48 \\
\pm 0.45\end{array}$ & $\begin{array}{l}2.37 \\
\pm 0.26\end{array}$ & $\begin{array}{r}1.87 \\
\pm 0.30\end{array}$ & $\begin{array}{r}1.83 \\
\pm 0.25\end{array}$ & $\begin{array}{l}2.38 \\
\pm 0.39\end{array}$ & $\begin{array}{l}2.19 \\
\pm 0.15\end{array}$ & & $\begin{array}{l}2.31 \\
\pm 0.36\end{array}$ & $\begin{array}{c}3.04 \\
\pm 0.69\end{array}$ & $\begin{array}{l}2.27 \\
\pm 0.28\end{array}$ & $\begin{array}{r}3.80 \\
\pm 0.81\end{array}$ & $\begin{array}{r}3.37 \\
\pm 0.43\end{array}$ & $\begin{array}{r}2.96 \\
\pm 0.25\end{array}$ & & $\begin{array}{l}4.07 \\
\pm 0.88\end{array}$ & $\begin{array}{c}2.57 \\
\pm 0.28\end{array}$ & $\begin{array}{c}3.79 \\
\pm 0.44\end{array}$ & $\begin{array}{c}3.48 \\
\pm 0.66\end{array}$ & $\begin{array}{r}3.73 \\
\pm 0.72\end{array}$ & $\begin{array}{c}3.53 \\
\pm 0.28\end{array}$ & \\
\hline \multirow{5}{*}{$\begin{array}{c}\mathrm{T}_{4} \\
\mathrm{pmol} / \mathrm{L}\end{array}$} & SWM & $\begin{array}{l}12.22 \\
\pm 1.73\end{array}$ & $\begin{array}{r}13.18 \\
\pm 2.59\end{array}$ & $\begin{array}{l}11.25 \\
\pm 2.13\end{array}$ & $\begin{array}{c}4.68 \\
\pm 0.98\end{array}$ & $\begin{array}{r}8.48 \\
\pm 2.25\end{array}$ & $\begin{array}{c}9.96 \\
\pm 1.01\end{array}$ & \multirow{5}{*}{$\begin{array}{c}\text { S }(0.000) \\
\text { HT }(0.001) \\
\text { S*HT } \\
(0.022)\end{array}$} & $\begin{array}{l}21.25 \\
\pm 5.22\end{array}$ & $\begin{array}{l}10.86 \\
\pm 1.86\end{array}$ & $\begin{array}{l}10.91 \\
\pm 1.80\end{array}$ & $\begin{array}{l}11.19 \\
\pm 2.03\end{array}$ & $\begin{array}{c}18.62 \pm 2 \\
.90\end{array}$ & $\begin{array}{l}14.56 \\
\pm 1.51\end{array}$ & \multirow{5}{*}{$\begin{array}{c}\text { S }(0.010) \\
\text { HT }(0.056) \\
\text { S*HT } \\
(0.031)\end{array}$} & $\begin{array}{r}11.23 \\
\pm 2.29\end{array}$ & $\begin{array}{l}13.04 \\
\pm 2.09\end{array}$ & $\begin{array}{l}15.73 \\
\pm 4.55\end{array}$ & $\begin{array}{l}11.40 \\
\pm 2.81\end{array}$ & $\begin{array}{l}11.23 \\
\pm 2.09\end{array}$ & $\begin{array}{r}12.52 \\
\pm 1.25\end{array}$ & \multirow{5}{*}{$\begin{array}{c}\mathrm{S}(0.001) \\
\mathrm{HT}(0.868) \\
\text { S*HT } \\
(0.602)\end{array}$} \\
\hline & NEM & $\begin{array}{c}6.67 \\
\pm 0.85\end{array}$ & $\begin{array}{l}12.11 \\
\pm 1.51\end{array}$ & $\begin{array}{c}7.41 \\
\pm 0.99\end{array}$ & $\begin{array}{c}9.26 \\
\pm 2.00\end{array}$ & $\begin{array}{c}9.36 \\
\pm 1.93\end{array}$ & $\begin{array}{r}8.96 \\
\pm 0.72\end{array}$ & & $\begin{array}{l}11.95 \\
\pm 3.21\end{array}$ & $\begin{array}{c}8.26 \\
\pm 1.31\end{array}$ & $\begin{array}{r}8.38 \\
\pm 0.67\end{array}$ & $\begin{array}{l}12.45 \\
\pm 4.13\end{array}$ & $\begin{array}{r}9.99 \\
\pm 1.56\end{array}$ & $\begin{array}{l}10.21 \\
\pm 1.10\end{array}$ & & $\begin{array}{l}12.41 \\
\pm 1.62\end{array}$ & $\begin{array}{c}8.67 \\
\pm 1.64\end{array}$ & $\begin{array}{r}9.66 \\
\pm 1.71\end{array}$ & $\begin{array}{l}14.54 \\
\pm 1.36\end{array}$ & $\begin{array}{l}14.57 \\
\pm 2.36\end{array}$ & $\begin{array}{l}11.97 \\
\pm 0.86\end{array}$ & \\
\hline & Winter & $\begin{array}{c}12.68 \pm 2.2 \\
1\end{array}$ & $\begin{array}{l}13.75 \\
\pm 2.33\end{array}$ & $\begin{array}{l}11.82 \\
\pm 2.45\end{array}$ & $\begin{array}{l}12.22 \\
\pm 1.59\end{array}$ & $\begin{array}{r}7.45 \\
\pm 1.41\end{array}$ & $\begin{array}{l}11.58 \\
\pm 0.94\end{array}$ & & $\begin{array}{l}14.29 \\
\pm 3.93\end{array}$ & $\begin{array}{l}21.92 \\
\pm 4.58\end{array}$ & $\begin{array}{r}9.87 \\
\pm 0.72\end{array}$ & $\begin{array}{l}16.63 \\
\pm 4.00\end{array}$ & $\begin{array}{c}15.72 \pm 2 \\
.26\end{array}$ & $\begin{array}{r}15.69 \\
\pm 1.59\end{array}$ & & $\begin{array}{r}18.09 \\
\pm 3.43\end{array}$ & $\begin{array}{l}18.58 \\
\pm 4.45\end{array}$ & $\begin{array}{l}13.34 \\
\pm 1.06\end{array}$ & $\begin{array}{l}16.12 \\
\pm 3.47\end{array}$ & $\begin{array}{l}16.70 \\
\pm 3.23\end{array}$ & $\begin{array}{l}16.57 \\
\pm 1.42\end{array}$ & \\
\hline & Summer & $\begin{array}{l}12.04 \\
\pm 1.95\end{array}$ & $\begin{array}{l}24.15 \\
\pm 2.82\end{array}$ & $\begin{array}{l}10.91 \\
\pm 0.93\end{array}$ & $\begin{array}{l}13.56 \\
\pm 2.50\end{array}$ & $\begin{array}{l}17.21 \\
\pm 3.74\end{array}$ & $\begin{array}{l}15.57 \\
\pm 1.38\end{array}$ & & $\begin{array}{l}12.54 \\
\pm 2.39\end{array}$ & $\begin{array}{l}12.89 \\
\pm 0.76\end{array}$ & $\begin{array}{l}12.17 \\
\pm 2.03\end{array}$ & $\begin{array}{l}23.00 \\
\pm 3.31\end{array}$ & $\begin{array}{c}16.47 \pm 2 \\
.19\end{array}$ & $\begin{array}{l}15.41 \\
\pm 1.21\end{array}$ & & $\begin{array}{l}15.12 \\
\pm 0.99\end{array}$ & $\begin{array}{l}16.45 \\
\pm 2.86\end{array}$ & $\begin{array}{l}21.16 \\
\pm 4.48\end{array}$ & $\begin{array}{l}18.39 \\
\pm 3.25\end{array}$ & $\begin{array}{l}22.32 \\
\pm 4.45\end{array}$ & $\begin{array}{l}18.69 \\
\pm 1.52\end{array}$ & \\
\hline & Total & $\begin{array}{l}10.90^{\mathrm{b}} \\
\pm 0.96\end{array}$ & $\begin{array}{c}15.80^{\text {acd }} \\
\pm 1.50\end{array}$ & $\begin{array}{l}10.35^{\mathrm{b}} \\
\pm 0.89\end{array}$ & $\begin{array}{l}9.93^{b} \\
\pm 1.11\end{array}$ & $\begin{array}{l}10.62 \\
\pm 1.41\end{array}$ & $\begin{array}{l}11.52 \\
\pm 0.56\end{array}$ & & $\begin{array}{l}15.01 \\
\pm 1.94\end{array}$ & $\begin{array}{l}13.48 \\
\pm 1.61\end{array}$ & $\begin{array}{l}10.33 \\
\pm 0.73\end{array}$ & $\begin{array}{c}15.81 \pm 1 \\
.88\end{array}$ & $\begin{array}{c}15.20 \pm 1 \\
.25\end{array}$ & $\begin{array}{l}13.97 \\
\pm 0.70\end{array}$ & & $\begin{array}{l}14.21 \\
\pm 1.19\end{array}$ & $\begin{array}{l}14.19 \\
\pm 1.58\end{array}$ & $\begin{array}{r}14.97 \\
\pm 1.78\end{array}$ & $\begin{array}{l}15.11 \\
\pm 1.42\end{array}$ & $\begin{array}{l}16.20 \\
\pm 1.70\end{array}$ & $\begin{array}{l}14.94 \\
\pm 0.68\end{array}$ & \\
\hline
\end{tabular}

S. Season, HT - Housing type, S*HT - Season verses Housing interaction. Figures in parenthesis indicates significance 
There was no significant difference for ChE in the interaction between the season and housing systems in all zones. Highest value of $\mathrm{ChE}$ (U/L) was found in metal housing in Southern zone $(670.7 \pm 130.8 \mathrm{U} / \mathrm{L})$ during summer and the lowest under open housing in High rainfall zone (56.9 $\pm 16.3 \mathrm{U} / \mathrm{L})$ during North East monsoon. Moreover, ChE activity was more in all the housing system. The increase in ChE activity results in the release of the neurotransmitter acetylcholine, which in turn alleviates the heat stress by promoting vasodilatation (Manabu Shibasaki et al., 2002).

\section{Lactate Dehydrogenase (LDH)}

Activity of LDH enzyme (LDH) was found to differ significantly $(\mathrm{P}<0.01)$ between seasons in all the zones. Significant difference $(\mathrm{P}<0.01)$ was observed for LDH between the housing systems in Southern and Cauvery delta zones. There was significant difference $(\mathrm{P}<0.01)$ for $\mathrm{LDH}$ in the interaction between the season and housing systems in Southern and Cauvery delta zones. Highest value of LDH (U/L) was found in tiled housing in Southern zone (2391.4 \pm 116.5 $\mathrm{U} / \mathrm{L}$ ) during winter and the lowest was found in metal housing in Cauvery delta zone (520.7 $\pm 89.8 \mathrm{U} / \mathrm{L})$ during summer. This findings were similar and in close agreement with the findings of Nazifi et al., (1999). The possible reason for the increased activity of $\mathrm{LDH}$ in Southern zone may be due to fast muscle twitching activity initiated from the body system during winter to maintain homeothermy. Moreover, in Southern zone only, lowest minimum temperature was recorded during winter. LDH is a ubiquitous enzyme with a number of iso-enzymes. Electrophoretic separation and estimation of iso-enzyme is necessary to arrive at a more finite conclusion. Normal serum levels of total LDH in cattle ranged from 692-1445 U/L

\section{Serum hormones}

The concentration of the three stress hormones viz. Cortisol, $\mathrm{T}_{3}$ and $\mathrm{T}_{4}$ have been determined in serum of animals under various housing types in all the four seasons of the three agro-climatic zones under study and are presented in Table 3.

\section{Cortisol}

Significant difference $(\mathrm{P}<0.01)$ in the concentration of cortisol level was observed between the seasons in all zones. Significant difference $(\mathrm{P}<0.05)$ in the concentration of cortisol between the housing types was recorded in Southern zone alone. No significant difference was observed for Cortisol in the interaction between the season and housing systems in all zones. Cortisol value $(\mu \mathrm{g} / \mathrm{dl})$ was found higher $(35.306 \pm 6.04 \mu \mathrm{g} / \mathrm{dl})$ in metal housing during winter season in High rainfall zone and the lower value was recorded $(1.895 \pm$ $0.33 \mu \mathrm{g} / \mathrm{dl}$ ) in tiled housing during summer in Southern zone. The concentration of cortisol was found to be significantly higher than the normal range $(0.61 \mu \mathrm{g} / \mathrm{dl})$ in all the climatic zones, in various housing types and different seasons studied (Hays FL et.al.1975).

\section{$\mathrm{T}_{3}$ hormone}

Significant difference $(\mathrm{P}<0.01)$ in the concentration of $T_{3}$ was observed between the seasons in all the zones. No significant difference was seen in the concentration of $T_{3}$ between the housing types in all the zones. There was no significant difference for $T_{3}$ in the interaction between the season and housing systems in all the zones. $\mathrm{T}_{3}$ value $(\mathrm{ng} / \mathrm{dl})$ was found higher $(6.92 \pm 2.38 \mathrm{ng} / \mathrm{dl})$ in thatched housing during North East monsoon season in High rainfall zone and the lower value was recorded $(0.61 \pm 0.10 \mathrm{ng} / \mathrm{dl})$ in metal housing during summer in High rainfall zone. Similar findings were reported by Magdub et al., (1982); they reported that plasma concentration of Triiodothyronine $\left(\mathrm{T}_{3}\right)$ in heat stressed lactating cows reduced significantly compared to animals kept in thermo neutral zone.

\section{$\mathrm{T}_{4}$ hormone}

There was significant difference $(\mathrm{P}<0.01)$ in the concentration of $\mathrm{T}_{4}$ between the seasons in all 
zones. $\mathrm{T}_{4}$ value (pmol/L.) was found higher $(24.15 \pm 2.82 \mathrm{pmol} / \mathrm{L}$.) in tiled housing during summer in Southern zone and the lower value was recorded $(4.68 \pm 0.98 \mathrm{pmol} / \mathrm{L})$ in metal housing during South West monsoon in Southern zone. Increased THI values results in decreased $\mathrm{T}_{4}$ during South West Monsoon. This was in close agreement with the findings of Bouraoui et al., (2002); they reported that when THI increased from 68 to 78 the free thyroxine concentration decreased from 15.5 to 14.5 pmol/L.

\section{References}

Bergmeyer, H.U. 1983. Methods of enzyme analysis. $3^{\text {rd }}$ edn., Verlogchemia, New York. Pp.133-138.

Bhooshan, N., K. Puneet, S.K, Singh and M.C. Yadav, 2010. Status of thyroid hormones in blood plasma of goats at different ages and their correlation with other biochemical parameters. Indian J. Anim. Sci. 80(7): 634-637.

Bouraoui, R., B. M. Lahmar, A. Majdoub, M. Djemali, and R. Belyea, 2002. The relationship of temperature-humidity index with milk production of dairy cows in a Mediterranean climate. Anim. Res. 51: 479-491.

Grunwaldt, E.G., J.C. Guevara, O.R. Estevez, A. Vicente, H. Rousselle, N. Alcuten, D. Aguerregaray, and C.R. Stasi, 2005. Biochemical and haematological measurements in beef cattle in Mendoza plain rangelands (Argentina). Trop. Anim. Health and Prod. 37(6): 527-540.
Hays, F.L. H. Armbruster, W. Vetter and W. Bianca 1975. Plasma cortisol in cattle: Circadian rhythm and exposure to a simulated high altitude of $5000 \mathrm{~m}$. International Journal of Biometeorology, 19 (2), 127-135

Kaneko, J.J., J.W. Harvey, and M.L. Bruss, 2008. Clinical biochemistry of domestic animals. $6^{\text {th }}$ ed., Academic Press, San Diego, CA, USA. pp. 882-887.

Magdub, A., H. D. Johnson, and R. L. Belyea, 1982. Effect of environmental heat and dietary fiber on thyroid physiology of lactating cows. J. Dairy Sci.65: 2323.

Manabu Shibasaki, Thad E. Wilson, Jian Cui and Craig G. Crandall 2002. Acetylcholine released from cholinergic nerves contributes to cutaneous vasodilation during heat stress. Journal of Applied Physiology, 93(6), 1947-1951.

Nazifi, S., H.R. Gheisari, and H. Poorabbas, 1999. The influences of thermal stress on serum biochemical parameters of dromedary camels and their correlation with thyroid activity. Comparative Haematology International. 9(1): 49-53.

Srikandakumar, A., and E.H. Johnson, 2004. Effect of Heat Stress on Milk Production, Rectal Temperature, Respiratory Rate and Blood Chemistry in Holstein, Jersey and Australian Milking Zebu Cows. Trop. Anim. Health Prod. 36(7): 685-692.

Whitaker, D.A., 2000. Use and interpretation of metabolic profiles. In: A.H. Andrews (ed.). The health of dairy cattle, Blackwell Science, Oxford, UK, 89-107

\section{How to cite this article:}

Paramasivam, A., P.S.L. Sesh, T. Sivakumar, P. Tensingh Gnanaraj and Thangavel, A. 2018. Impact of Different Shelter Designs on Selected Serum Enzyme Activities and on Concentration of Stress Hormones in Dairy Cattle of Tamil Nadu. Int.J.Curr.Microbiol.App.Sci. 7(01): 886-893. doi: https://doi.org/10.20546/ijcmas.2018.701.108 